\title{
All-Optical Processing of Multi-level Phase Shift Keyed Signals
}

\author{
Joseph Kakande*, Radan Slavík, Francesca Parmigiani, Periklis Petropoulos and David J. Richardson \\ Optoelectronics Research Centre, University of Southampton, Southampton, SO17 1BJ, UK \\ *jkk@orc.soton.ac.uk
}

\begin{abstract}
A technique that allows the all-optical quantization of M-PSK signals using a parametric mixing process is discussed, including recent experimental demonstrations of high baud rate QPSK regeneration, and issues regarding future scalability of the scheme.

OCIS codes: (060.2320) Fibre optics amplifiers and oscillators, (190.4410) Nonlinear optics, parametric processes
\end{abstract}

\section{Introduction}

For optical networks to continue meeting the insatiable consumer demand for transmission bandwidth, efficiencies must be delivered in both use of the finite utilizable spectrum, as well as in power usage. The rapid growth of the internet has led to estimates that by 2025 the internet will consume $7 \%$ of the 2010 global electricity supply [1]; in that same period extrapolating current $1.5 \mathrm{~dB} / \mathrm{yr}$ traffic growth rates (Cisco visual networking index: forecast and methodology, 2010-2015) suggests that capacity must grow by a factor exceeding 100. The ability of all-optical techniques to out-compete optical-to-electrical-to-optical (OEO) conversion alternatives in terms of raw speed, particularly when it comes to simple functions such as high-speed switching is universally acknowledged. In energy terms however, advances in semiconductor electronics mean that OEO based switches are currently often more efficient than their all-optical counterparts, once the power consumption of both the switch and ancillary devices (such as optical pump amplifiers for many Kerr-based devices) is taken into account [1].

All-optical signal processing devices using Kerr nonlinearity have been shown to be capable of handling legacy modulation formats $[2,3]$, although to date there has been very little commercial deployment. While OEO devices acting on single bit/symbol inputs perform well enough in energetic terms to render optical equivalents uncompetitive, the situation could be reversed with the advent of complex modulation formats. A fully coherent regenerator for a single polarization QPSK signal would require a local oscillator, dual channel high speed analogto-digital converter (ADC), a high speed digital signal processing (DSP) chip, and a QPSK transmitter. For a 25 Gbaud DSP-based QPSK regenerator, the 25 optical Gsymbols/s received would be transformed to two $150 \mathrm{Gbit} / \mathrm{s}$ serial electrical streams due to the requirement of at least $6 \mathrm{bit} / \mathrm{symbol}$ resolution in the ADCs for optimal performance [4]. As a result, an electrical regenerator would need to process at least 6 times as many symbols as an equivalent analog (all-optical) device that directly regenerated each received symbol. This internal symbolmultiplication, inherent to coherent OEO regenerators, may make it possible for all-optical regenerators to become competitive, as it overcomes the per-bit switching disadvantage [1] relative to OEO approaches.

Here, an all-optical phase quantizer is described that is able to reduce phase fluctuations in M-PSK signals. This utilizes ultra-fast four wave mixing (FWM), allowing for almost unlimited processing speed, as well as the ability to rapidly switch the quantization function to match the input M-PSK signal, a flexibility usually restricted to electronic, rather than optical technologies. The concept has been experimentally verified [5, 6], and some further issues regarding practicality and scalability are addressed here.

\section{Concept and demonstration}

From an arbitrary phase encoded signal $\exp (i \varphi(\mathrm{t}))$, we can synthesize an M-level phase quantized output $\exp \left(i \varphi_{S}(t)\right)$ by coherently adding to the signal a phase conjugated harmonic bearing a temporal phase modulation M-1 times that on the signal. The quantization function is shown in Eqn. 1. The phase quantization is accompanied by a sinusoidal amplitude response $A_{S}$ of periodicity $2 \pi / \mathrm{M}$.

$$
\begin{gathered}
\left|A_{S}\right| \cdot \exp \left(i \varphi_{S}(t)\right)=\exp (i \varphi(\mathrm{t}))+m \cdot \exp (-i \varphi(\mathrm{t}) \cdot(M-1)) \\
\text { Misfit Factor }=\log \left(\frac{2}{\pi} \cdot \int_{\frac{-\pi}{M}}^{\frac{\pi}{M}}\left|\varphi_{S}(m, \varphi)\right| d \varphi\right)
\end{gathered}
$$

Figs. 1a and $1 \mathrm{~b}$ show the phase and amplitude transfer functions respectively as $m$ in Eqn. 1 is varied for $M=4$ (QPSK). To systematically find the most suitable value, a misfit factor was designed such that an optimisation process 
could be numerically performed. The misfit factor, as calculated in Eqn. 2 integrates the difference between the achieved phase transfer function and an ideal stepped function. The optimisation is then done by finding a value of $m$ that minimises the misfit factor. Fig 1c shows the calculated optimal $m$ values for 2 to 16 -PSK.
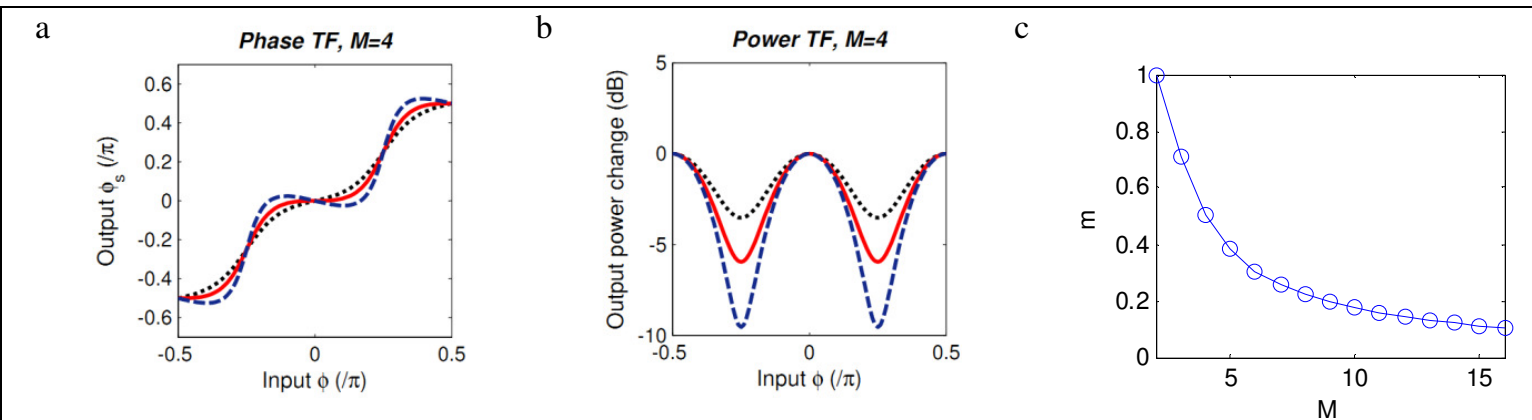

Fig. $1(\mathrm{a}, \mathrm{b})$ Evaluation of quantization transfer function (TF) in Eqn. 1 for $\mathrm{M}=4$, showing the phase and amplitude transfer functions for various values of $m$. Dotted line is for $m=0.25$, solid line $m=0.33$, and dashed line $m=0.5$. (c) Optimal $m$ value as M is varied, located using misfit factor analysis in Eqn. 2.

Experimentally, the operations of phase multiplication, conjugation and coherent addition are performed using FWM. First, the phase harmonic is generated from the signal using a cascaded FWM process in a highly nonlinear fibre (HNLF), which is performed by combining the signal with a strong frequency detuned pump in a nonlinear medium. By optimising the phase matching and the strength of the nonlinear interaction, a spectral cascade of FWM harmonics are generated [6]. Because FWM is momentum conserving, the harmonics possess an overlying phase modulation that is a perfect integer multiple of the modulation present on the signal at the mixer input. Next, a second FWM process is carried out using two pumps located symmetrically around the signal and phase harmonic to coherently conjugate and add the phase harmonic to the signal. By careful choice of fibre and operating wavelengths, both these functions can be obtained in a distributed manner in a single fibre [5]. Note that high SBS threshold fibers are required to use high power, narrow linewidth pumps in the mixing process. The results shown here were achieved with an alumino-silicate fibre from OFS Denmark [7], one of the many advances in optical devices enabling today's sophisticated all-optical subsystems.

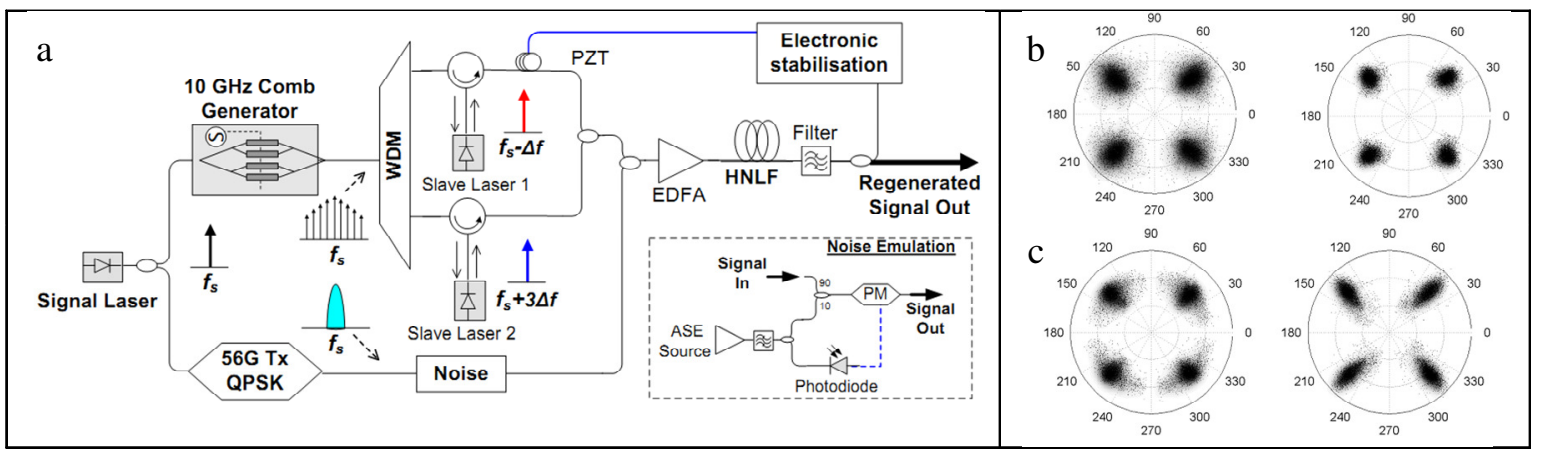

Fig. 2 (a) Demonstration of QPSK regeneration, taken from [5]. A QPSK signal was mixed with two phase locked pumps synthesized from an optical comb. WDM-wavelength division multiplexer, PZT - piezoelectric fiber stretcher, Tx - transmitter , ASE - amplified spontaneous emission, PM - phase modulator. (b) input and output constellations for a 10 Gbaud signal degraded in amplitude and phase. (c) input and output constellations for a 56 Gbaud signal degraded by strong phase noise.

The quantizer was set up and tested as a QPSK regenerator, as described in [5]. Various levels of noise in phase and amplitude were added to an input QPSK signal, and the signal quality before and after the regenerator was assessed using an EXFO constellation analyzer (PSO-200), based on all-optical sampling and capable of operation up to 100 Gbaud. At 10 Gbaud, and in the presence of linear noise emulated by ASE loading (hence degraded OSNR with both phase and amplitude fluctuations), the phase error variance and normalised amplitude variance were simultaneously reduced by approximately 3.2. The symbol rate was increased to 56 Gbaud and the device tested, this time with a noise distribution closer emulating strong nonlinear phase noise. Again, the phase error variance was reduced by a factor of 3.2, albeit with increased amplitude noise at the output. For QPSK in which the information is solely contained in the phase, this nonlinear phase noise reduction could be used to increase reach, or to increase 
signal launch powers to improve OSNR at the receiver. Note that this functionality was obtained for total powers under $1 \mathrm{~W}$.

\section{Performance and scalability}

One key benefit of performing all optical phase quantization is that following optical correction of phase errors, a differential receiver can be used instead of a homodyne (or coherent) receiver, without a significant penalty. This is because the differential phase error distribution rapidly approaches the absolute error distribution after the quantizer, a phenomenon previously observed with a DPSK regenerator based on a degenerate FWM [8]. Numerical simulations (split step Fourier method) are used here to illustrate this effect.

In the simulation, a pseudo-random 8-PSK signal was generated at $80 \mathrm{Gbaud}$, as well as a harmonic idler $3 \mathrm{~nm}$ away whose phase modulation was 7 times that on the signal. The idler power was $13 \mathrm{~dB}$ less than that on the signal, this value calculated by squaring $m$ as calculated in Fig. 1c. The two waves were then mixed in a $250 \mathrm{~m}$ HNLF by a single $0.5 \mathrm{~W}$ pump located exactly halfway between the signal and idler. The fibre had nonlinear coefficient 11 $/ \mathrm{W} / \mathrm{km}$ and dispersion at the pump frequency of $0.01 \mathrm{ps} / \mathrm{nm} / \mathrm{km}$. Note how at the quantizer input the differential constellation is significantly worse than the absolute constellation, Figs. $3 \mathrm{a}$ and $3 \mathrm{~b}$ respectively. However, after the quantizer, the differential (Fig. 3c) and absolute (Fig. 3d) constellations have similar phase statistics, both of which have less phase deviations than the input absolute phase distribution. Perhaps this functionality can in future be used to deploy low cost, low power differential M-PSK systems without compromising on performance relative to fully coherent receivers.

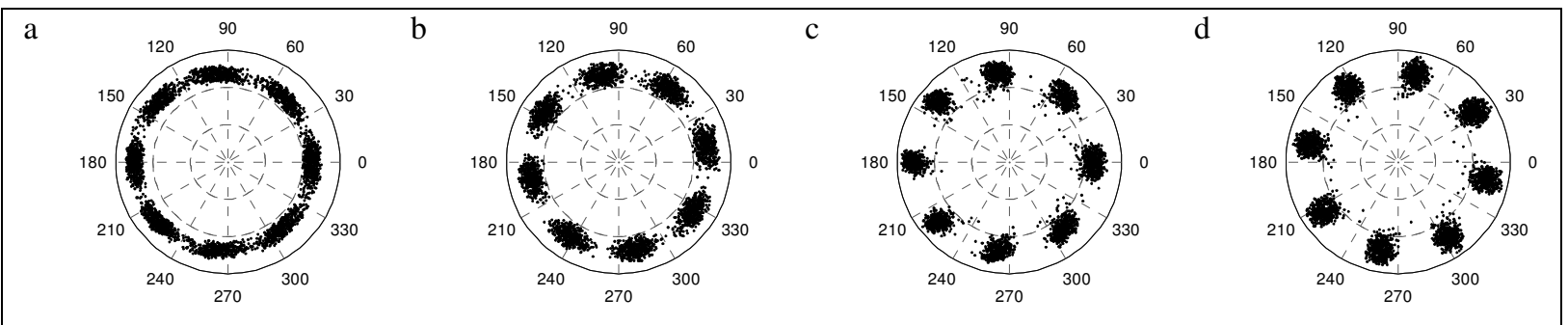

Fig. 3 Results of numerical simulation showing quantization effect for an 80 Gbaud 8-PSK signal. (a,b) input differential and absolute constellations respectively. (c,d) output differential and absolute constellations respectively.

\section{Conclusions}

The multilevel all-optical phase quantization concept, of combining an M-PSK signal with a selected phase harmonic, is extremely powerful due to the fact that a single device is used per input symbol, not per input bit. The device is capable of performing phase regeneration in an optical network, at modest optical powers, and the numerical and experimental evidence to date suggests that the scheme can cope with future denser phase encoded formats such as 8-PSK. An optimistic prediction would point towards advances in nonlinear media, in narrow linewidth phase locked pump technology, and in photonic integration enabling the quantizer to become a practical building block in a wide range of subsystems, from regenerators to all-optical ADCs.

\section{Acknowledgement}

We would like to thank to OFS Denmark for the HNLF; Eblana Photonics, Ireland for the discrete mode lasers; and Adonis Bogris (University of Athens) for useful discussions. This research has received funding from the European Communities Seventh Framework Program FP/2007-2013 under grant agreement 224547 (PHASORS).

\section{References}

[1] R. S. Tucker, "Green Optical Communications Part II: Energy Limitations in Networks," Selected Topics in Quantum Electronics, IEEE Journal of, vol. 17, pp. 261-274, 2011.

[2] P. V. Mamyshev, "All-optical data regeneration based on self-phase modulation effect," in Optical Communication, 1998. 24th European Conference on, 1998, pp. 475-476 vol.1.

[3] R. Slavík, et al., "All-optical phase and amplitude regenerator for next-generation telecommunications systems," Nat Photon, vol. 4, p p. 690-695, 2010.

[4] I. Dedic, "56Gs/s ADC : Enabling 100GbE," in Optical Fiber Communication (OFC), collocated National Fiber Optic Engineers Conference, 2010 Conference on (OFC/NFOEC), paper OThT6, 2010.

[5] J. Kakande, et al., "QPSK phase and amplitude regeneration at 56 Gbaud in a novel idler-free non-degenerate phase sensitive amplifier.," OFC 2011, Los Angeles, 6-10 Mar 2011 p. OMT4, 2011.

[6] J. Kakande, et al., "Multilevel quantization of optical phase in a novel coherent parametric mixer architecture," Nature Photonics, doi 10.1038/NPHOTON.2011.254, 2011.

[7] L. Gruner-Nielsen, et al., "Silica-based highly nonlinear fibers with a high SBS threshold," in Winter Topicals (WTM), 2011 IEEE, pp. 171-172.

[8] R. Slavík, et al., "Coherent All-Optical Phase and Amplitude Regenerator of Binary Phase-Encoded Signals," doi 10.1109/JSTQE.2011.2136329, 2011. 